\title{
REGULARIZED INTERPOLATION FOR NOISY DATA
}

\author{
Sathish Ramani, Philippe Thévenaz, Michael Unser \\ Biomedical Imaging Group, \\ École Polytechnique Fédérale de Lausanne (EPFL), \\ CH-1015 Lausanne VD, Switzerland.
}

\begin{abstract}
Interpolation is a vital tool in biomedical signal processing. Although there exists a substantial literature dedicated to noise-free conditions, much less is known in the presence of noise. Here, we document the breakdown of standard interpolation for noisy data and study the performance improvement due to regularized interpolation. In particular, we numerically investigate the Tikhonov (quadratic) regularization. On top of that, we explore non-quadratic regularization and show that this yields further improvements. We derive a novel bounded regularization approach to determine the optimal solution. We justify our claims with experimental results.
\end{abstract}

Index Terms - Interpolation, B-spline, Regularization, Tikhonov criterion, Total Variation, Bounded Optimization.

\section{INTRODUCTION}

Interpolation is an essential ingredient of biomedical applications such as resampling or registration, and sound theory and efficient methods have already been developed in the noisefree case [1]. When data is significantly corrupted by noise, however, the standard techniques fail. Tikhonov-regularized interpolation - quadratic functional - is a potential remedy that has been proposed in [2], but there it is restricted to the $1-\mathrm{D}$ case. In this work, we extend that solution to multiple dimensions for the specific case of the gradient operator, and experimentally verify its performance by comparing against standard (non-regularized) interpolation. In addition, we consider functionals based on the $L_{p}$ norm of a suitable differential operator with $1 \leq p \leq 2$. For $p \neq 2$, the solution requires a non-linear optimizer. We therefore propose a general bounded-optimization approach and use it to show experimentally that, in the presence of noise, non-quadratic regularization yields better interpolation as compared to the Tikhonov method.

\section{STANDARD INTERPOLATION}

Interpolation is the process of computing a continuously defined function $f_{\text {int }}(\mathbf{x}), \mathbf{x}=\left(x_{1}, x_{2}, \ldots, x_{d}\right) \in \mathbb{R}^{d}$ given samples $g[\mathbf{k}], \mathbf{k}=\left(k_{1}, k_{2}, \ldots, k_{d}\right) \in \mathbb{Z}^{d}$. The interpolant $f_{\text {int }}$ is chosen to belong to the functional space $V_{\varphi}$ defined as

$$
V_{\varphi}=\left\{s(\mathbf{x}): s(\mathbf{x})=\sum_{\mathbf{k} \in \mathbb{Z}^{d}} c[\mathbf{k}] \varphi(\mathbf{x}-\mathbf{k})\right\},
$$

where $\varphi$ is called the synthesis function [1]. For a given $\varphi$, it is desired to find the coefficients $\{c[\mathbf{k}]\}$ of $f_{\text {int }}$ such that $\left.f_{\text {int }}(\mathbf{x})\right|_{\mathbf{x}=\mathbf{k}}=g[\mathbf{k}]$. Up to technical details related to the choice of $\varphi$, the (unique) solution is given by $c[\mathbf{k}]=$ $\left(g * h_{\text {int }}\right)[\mathbf{k}]$. Thus, the coefficients can be directly obtained by convolving (linear filtering) the samples $\{g[\mathbf{k}]\}$ with the interpolation filter $h_{\text {int }}[\mathbf{k}]$ with frequency response

$$
H_{\mathrm{int}}\left(e^{j \boldsymbol{\omega}}\right)=\frac{1}{\sum_{\mathbf{k} \in \mathbb{Z}^{d}} \hat{\varphi}(\boldsymbol{\omega}+2 \pi \mathbf{k})},
$$

where $\hat{\varphi}(\boldsymbol{\omega})$ for $\boldsymbol{\omega} \in \mathbb{R}^{d}$ is the Fourier transform of $\varphi$.

\subsection{Interpolation in the Presence of Noise}

According to the theory of approximation, the higher the order of approximation of $\varphi$, the better the quality of interpolation. Unfortunately, the presence of noise violates the assumptions of this approximation-theoretic argument because the signal is not deterministic anymore. The imperfections caused by interpolating noisy samples nevertheless still depend on the behavior of the corresponding interpolator $\varphi_{\text {int }}$ which has the Fourier transform $\hat{\varphi}_{\text {int }}(\boldsymbol{\omega})=H_{\text {int }}\left(e^{j \boldsymbol{\omega}}\right) \hat{\varphi}(\boldsymbol{\omega})$.

Consider now the following experiment: First rotate some input image using a high-quality interpolator. Then, add the proper amount of zero-mean white Gaussian noise to obtain a prescribed signal-to-noise ratio (SNR). To avoid boundary artifacts, compute all SNRs over a circular region concentric with the image. Finally, to compare linear and cubic B-spline interpolation, rotate back the noisy image using either method to get the final (output) image. We have plotted the SNR of the output image in Figure 1. In contradiction to approximationtheoretic arguments, we observe that linear outperforms cubic under noisy conditions. Qualitatively, this can be explained as follows: the Fourier transform of the cubic B-spline interpolator has a higher gain for certain high frequencies compared to that of the linear B-spline interpolator [3]. This implies that, for certain noise levels, spurious high frequencies will be amplified, which leads to a poor performance of cubic B-spline as shown in Figure 1.

\section{REGULARIZED INTERPOLATION}

Given noisy data $g[\mathbf{k}], \mathbf{k} \in \mathbb{Z}^{d}$, interpolation with regularization [2] aims to obtain a continuous space function $f \in V_{\varphi}$, that is "smooth" while ensuring that $f(\mathbf{k})$ are "close" to $g[\mathbf{k}]$. 


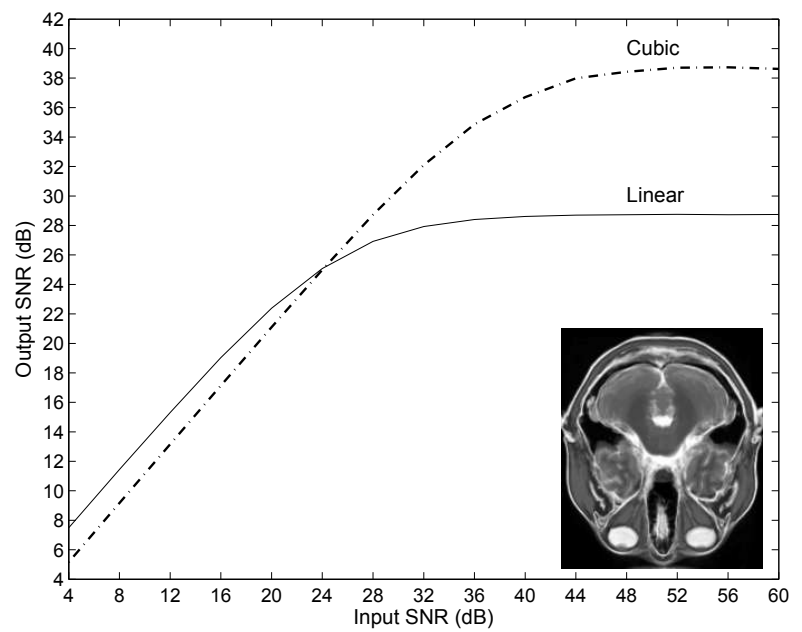

Fig. 1. Rotation experiment in the presence of noise: Failure of standard interpolation.

Mathematically, the problem is formulated as

$$
f_{\text {reg }}=\arg \min _{f \in V_{\varphi}} \mathcal{J}\{f\},
$$

where

$$
\mathcal{J}\{f\}=\|g[\mathbf{k}]-f(\mathbf{k})\|_{\ell_{2}}^{2}+\lambda \int_{\mathbb{R}^{d}}|\mathrm{~L}\{f\}(\mathbf{x})|^{p} \mathrm{~d} \mathbf{x} .
$$

Since, $f_{\text {reg }} \in V_{\varphi}$, (3) can be reformulated as

$$
f_{\text {reg }}(\mathbf{x})=\sum_{\mathbf{k} \in \mathbb{Z}^{d}} c_{\text {reg }}[\mathbf{k}] \varphi(\mathbf{x}-\mathbf{k})
$$

where

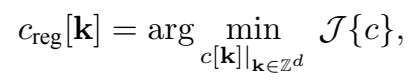

where $\mathcal{J}\{c\}$ is the cost given by

$$
\begin{aligned}
\mathcal{J}\{c\}= & \sum_{\mathbf{k}}|g[\mathbf{k}]-(c * b)[\mathbf{k}]|^{2} \\
& +\lambda \int_{\mathbb{R}^{d}}\left|\sum_{\mathbf{k} \in \mathbb{Z}^{d}} c[\mathbf{k}] \operatorname{L}\{\varphi\}(\mathbf{x}-\mathbf{k})\right|^{p} \mathrm{~d} \mathbf{x},
\end{aligned}
$$

where $1 \leq p \leq 2, b[\mathbf{k}]=\left.\varphi(\mathbf{x})\right|_{\mathbf{x}=\mathbf{k}}$, L is a suitable differential operator (e.g., Gradient or Laplacian), and $\lambda>0$ is the regularization parameter.

Thus, regularized interpolation (3) is similar to standard interpolation, except that now the coefficients are obtained from (6). Moreover, the relation between $\left\{c_{\text {reg }}[\mathbf{k}]\right\}$ and the samples $\{g[\mathbf{k}]\}$ depends on the value of $p$. It should also be noted that, as $\lambda \rightarrow 0$, the effect of regularization diminishes and $f_{\text {reg }}$ eventually performs standard interpolation of the noisy data $g[\mathbf{k}]$ (which is also the least-squares solution).

\subsection{Quadratic (Tikhonov) Functional}

Choosing $p=2$ in (7) leads to a quadratic regularization. In $1 \mathrm{D}$, the solution has been worked out in [2]. Here, we extend that solution to $d$ dimensions. We shall deal explicitly with the gradient operator henceforth, noting that a similar analysis applies to the Laplacian operator. Choosing $\mathrm{L}=\nabla$ and $p=2$, the solution to (6) can be obtained as $c[\mathbf{k}]=$ $\left(g * h_{\lambda}\right)[\mathbf{k}]$, which is linear in $\{g[\mathbf{k}]\}$, and where $h_{\lambda}[\mathbf{k}]$ is the digital-correction filter with frequency response

$$
\begin{gathered}
H_{\lambda}\left(e^{j \boldsymbol{\omega}}\right)=\sum_{\mathbf{k} \in \mathbb{Z}^{d}} \hat{\varphi}^{\star}(\boldsymbol{\omega}+2 \pi \mathbf{k})\left(\left|\sum_{\mathbf{k} \in \mathbb{Z}^{d}} \hat{\varphi}(\boldsymbol{\omega}+2 \pi \mathbf{k})\right|^{2}\right. \\
\left.+\lambda \sum_{i=1}^{d} \sum_{\mathbf{k} \in \mathbb{Z}^{d}}\left|\omega_{i}+2 \pi k_{i}\right|^{2}|\hat{\varphi}(\boldsymbol{\omega}+2 \pi \mathbf{k})|^{2}\right)^{-1}
\end{gathered}
$$

where $\partial / \partial x_{i} \stackrel{\mathcal{F}}{\longleftrightarrow}\left(j \omega_{i}\right)$, and $\star$ represents the complex conjugate. As expected when $\lambda \rightarrow 0,(8)$ becomes $H_{\text {int }}\left(e^{j \boldsymbol{\omega}}\right)$ and we get back the standard interpolation filter. Besides, while $H_{\text {int }}$ can be separable $H_{\lambda}$ is never, for $\lambda>0$.

\subsection{Non-Quadratic (NQ) Functional}

For $1 \leq p<2$, the regularization in (7) is non-quadratic. The case $\mathrm{L}=\nabla$ and $p=1$ corresponds to the Total-Variation (TV) regularization introduced in [4], which has recently gained attention in deconvolution problems [5]. For $p \neq 2$, one immediately sees that the solution is nonlinear. Moreover, the integral in (7) does not have a closed form expression. Hence the regularization term must be discretized.

\subsubsection{Discretization}

We discretize the regularization term on the grid of the samples. For the 2-D case, using the fact that $u \in V_{\varphi}$, we have

$$
\begin{aligned}
\int_{\mathbb{R}^{2}}|\nabla f(\mathbf{x})|^{p} \mathrm{~d} \mathbf{x} & \approx \sum_{i, j}\left(\sqrt{\eta_{1}^{2}[i, j]+\eta_{2}^{2}[i, j]}\right)^{p} \\
& =R_{p}\left\{\eta_{1}, \eta_{2}\right\}
\end{aligned}
$$

where $\eta_{1}[i, j]=\left(c * \varphi_{x_{1}}\right)[i, j], \eta_{2}[i, j]=\left(c * \varphi_{x_{2}}\right)[i, j]$, and $\varphi_{x_{k}}[i, j]=\partial \varphi /\left.\partial x_{k}\right|_{x_{1}=i, x_{2}=j}$. Therefore, the cost to be minimized is

$$
\mathcal{J}_{\mathrm{NQ}}\{c\}=\sum_{i, j}|g[i, j]-(c * b)[i, j]|^{2}+\lambda R_{p}\left\{\eta_{1}, \eta_{2}\right\} .
$$

\subsubsection{Minimization}

We resort to the bounded-optimization method (also called the Majorization-Minimization(MM) method) which has also been recently applied to TV deconvolution [5]. In brief, we first bound $\mathcal{J}_{\mathrm{NQ}}\{c\}$ by a weighted-quadratic functional $\mathcal{J}_{\mathrm{WQ}}\{c\}$. Then, we repeatedly minimize $\mathcal{J}_{\text {WQ }}$ (which is easier than minimizing $\mathcal{J}_{\mathrm{NQ}}$ ) with updated weights to ensure the decrease of $\mathcal{J}_{\mathrm{NQ}}$.

To obtain $\mathcal{J}_{\mathrm{WQ}}$, it has been noted in [6] that

$$
|x|^{p} \leq \frac{2-p}{2}\left|x_{0}\right|^{p}+\frac{p}{2} x^{2}\left|x_{0}\right|^{p-2}
$$


where $1 \leq p<2$, for some $x_{0} \neq 0$, and where the equality holds only when $x=x_{0}$. As this inequality is preserved under summation and multiplication by a positive constant [6],

$$
\lambda R_{p}\left\{\eta_{1}, \eta_{2}\right\} \leq \lambda R_{\mathrm{WQ}}\{\theta\},
$$

where

$$
\begin{aligned}
R_{\mathrm{WQ}}\{\theta\}= & \frac{2-p}{2} \sum_{i, j=1}^{N}|\theta[i, j]|^{p} \\
& +\frac{p}{2} \sum_{i, j=1}^{N}\left(\eta_{1}^{2}[i, j]+\eta_{2}^{2}[i, j]\right)|\theta[i, j]|^{p-2},
\end{aligned}
$$

and where $\theta[i, j]=\sqrt{\eta_{1,0}^{2}[i, j]+\eta_{2,0}^{2}[i, j]}$ is obtained for some $\left\{c_{0}[i, j]\right\}$ in (9). Thus, for a given $\{\theta[i, j]\}, \mathcal{J}_{\mathrm{NQ}}\{c\}$ is bounded above as

$$
\mathcal{J}_{\mathrm{NQ}}\{c\} \leq \mathcal{J}_{\mathrm{WQ}}\{c, \theta\},
$$

where

$$
\mathcal{J}_{\mathrm{WQ}}\{c, \theta\}=\sum_{i, j=1}^{N}|g[i, j]-(c * b)[i, j]|^{2}+\lambda R_{\mathrm{WQ}}\{\theta\} .
$$

Then, (13) leads to the following MM-algorithm:

Step 1: Initial estimate $=\left\{c_{0}[i, j]\right\} ; t=0$

Repeat Steps 2 and 3 until Stop Criterion is met

Step 2: Update $\left\{\theta_{t}[i, j]\right\}$ using $\left\{c_{t}[i, j]\right\}$; Set $t=t+1$

Step 3: Minimize $\mathcal{J}_{\mathrm{WQ}}\left\{c_{t}, \theta_{t-1}\right\}$ to obtain $\left\{c_{t}[i, j]\right\}$

Since $\mathcal{J}_{\mathrm{WQ}}\left\{c_{t}, \theta_{t-1}\right\}$ is quadratic, its minimization can be done in many ways, for instance, either by solving a linear system of equations or by the conjugate-gradient (CG) method. The latter is a widely used method whenever the size of the signal is very large (especially images). We then update $\left\{\theta_{t}[i, j]\right\}$ using the current $\left\{c_{t}[i, j]\right\}$. Repeating this process ensures that $\mathcal{J}_{\mathrm{NQ}}$ is reduced at each iteration because of the inequality (13). This method is also called the re-weighted least squares (RWLS) because the weights $\{\theta[i, j]\}$ are updated repeatedly each time we minimize $\mathcal{J}_{\mathrm{WQ}}\left\{c_{t}, \theta_{t-1}\right\}$. In this case the proposed algorithm is exactly the same as the multiplicative form of the half-quadratic method [7] with the auxiliary variable $|\theta[i, j]|^{p-2}$, the dual function $\sum_{i, j=1}^{N} \frac{2-p}{2}|\theta[i, j]|^{p}$, and with steps 2 and 3 forming the alternate minimization sequence.

\section{EXPERIMENTS}

Here, we choose $\varphi(\mathbf{x})=\beta^{3}\left(x_{1}\right) \beta^{3}\left(x_{2}\right)$ (the separable cubic B-spline) for regularized interpolation and the TV functional for the non-quadratic regularization. To test the performance of the Tikhonov-regularized cubic-spline and TVregularized cubic-spline interpolation, we perform rotation experiments similar to those described in Section 2.1. The optimal $\lambda$ was found empirically for both methods. They were then compared with standard interpolation techniques such as nearest-neighbor interpolation, standard linear interpolation, and standard cubic interpolation [1].

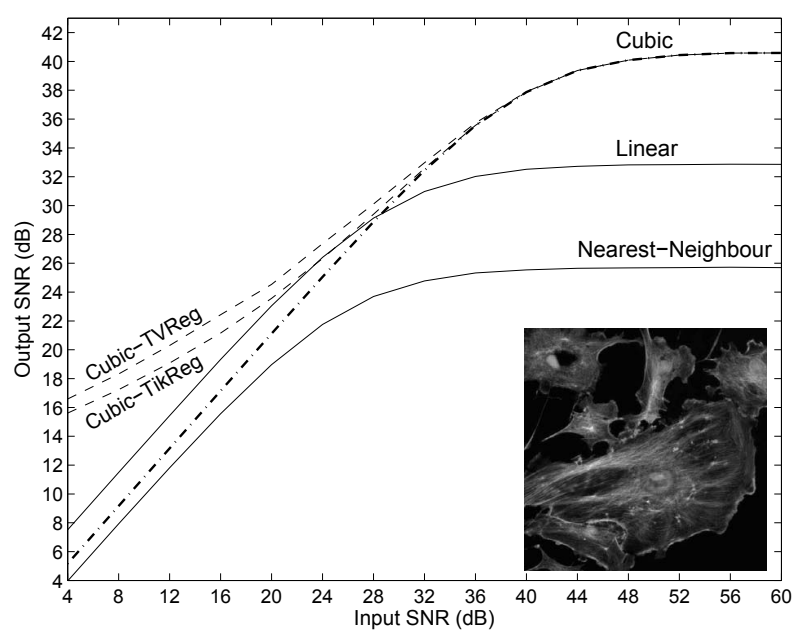

Fig. 2. SNR comparison for Experiment 1: Single (Fluorescence) image.

\subsection{Single Image}

We make use of the $(512 \times 512)$ biological specimen shown in the inset of Figure 2 for this experiment. For a given input SNR, we perform 25 rotation experiments with a rotation angle drawn at random from $[0,2 \pi)$ and averaged the output SNR over these 25 realizations. We then repeat this for different input SNR values. We show in Figure 2 the resulting plots.

Several observations are in order. Firstly, we note that the nearest-neighbor method always performs poorly, which is quite expected. Secondly, for input SNRs below $28 \mathrm{~dB}$, linear interpolation does better than cubic B-spline, a behavior that we already discussed in Section 2.1. The Tikhonovregularized method performs better than any of the non-regularized ones. However, it tends to blur edges (see Figure 3); hence, it is not as effective as the TV-regularized interpolation which always outperforms all methods. This indicates that TV-regularized interpolation is more sturdy.

Another significant observation is that the SNR of the two regularized methods (for cubic B-spline) converge to that of the standard cubic B-spline for relatively high input SNRs. This is to be expected because, under very low noise, the effect of regularization is negligible. Moreover, all the methods saturate beyond a particular input SNR because of the inherent degradation caused by the very process of interpolation.

\subsection{Multiple Images}

We want now to test the performance of the methods on different images. For this, we obtained from [8] a stack of clean MRI images (T1, T2, PB) with uniform intensity and $9 \mathrm{~mm}$ slice thickness. We zero-padded the images to size $(300 \times 300)$ to provide margins for the rotations. For a given input SNR, we perform 100 rotation experiments; for each one, we pick at random a slice from the stack and rotate it by a random angle that is uniformly distributed over $[0,2 \pi)$. We then average the output SNR over the 100 realizations. This was repeated for different input SNRs and lead to Figure 4.

The behavior is qualitatively similar to that found in Figure 2; the same explanations are valid here too. However, the 

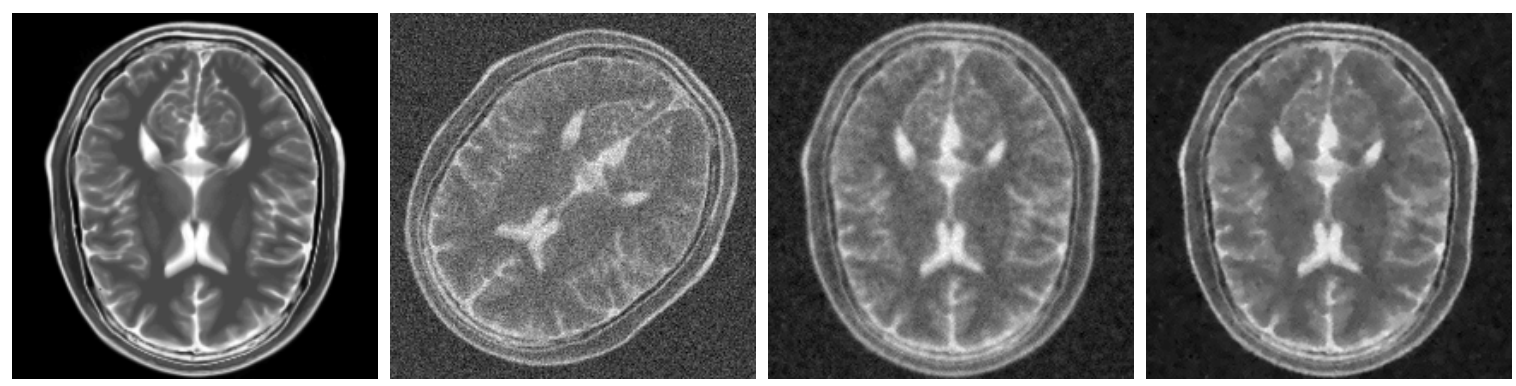

Fig. 3. A realization of the second experiment. From left to right: Original Image; Noisy-Rotated Image: rotation by $40^{\circ}, 11.68$ $\mathrm{dB}$; Tikhonov-regularized interpolation: $18.06 \mathrm{~dB}$; TV-Regularized interpolation: $20.261 \mathrm{~dB}$. The SNRs were computed over the set of non-zero pixels of the original image.

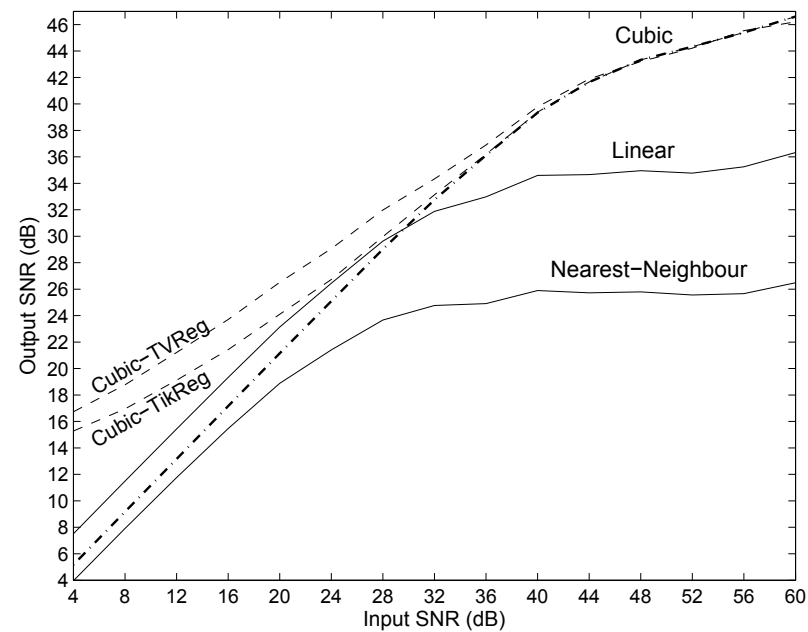

Fig. 4. SNR comparison for experiment 2: MRI Stack.

main point now is that the images used for the experiments are different from one another. A consistently superior behavior of TV-regularized method in this scenario clearly illustrates its robustness compared to the other methods. We present in Figure 3 one realization of the second experiment. As anticipated, the results for the Tikhonov-regularized interpolation appear blurred compared to the TV-regularized solution.

\section{SUMMARY, CONCLUSIONS, AND DISCUSSION}

Standard interpolation fails to work when signals are corrupted by excessive noise. The use of a quadratic functional (Tikhonov-regularized interpolation) can improve the situation but compromises the sharpness of the output. In this work, we have proposed the use of a non-quadratic functional such as TV (which preserves sharp features) for regularized interpolation. We have experimentally quantified the improvement in performance. We conclude that regularization based on non-quadratic functionals (such as TV) yield better results compared to the Tikhonov regularized method.

Our main concern here is to highlight the improved performance of regularized interpolation; we did skip over finer details such as choosing the best $\lambda$ since significant literature is already dedicated to this problem [9]. Of greater interest is the fact that regularized interpolation does not end with the case of Gaussian noise. Instead, by choosing the log- likelihood corresponding to the noise distribution as the data term, and by designing a suitable algorithm, our future work is to extend this formulation to data corrupted by Poisson and Speckle noise.

\section{REFERENCES}

[1] P. Thévenaz, T. Blu, and M. Unser, "Image interpolation and resampling," in Handbook of Medical Imaging, Processing and Analysis, I.N. Bankman, Ed., chapter 25, pp. 393-420. Academic Press, San Diego CA, USA, 2000.

[2] Y.C. Eldar and M. Unser, "Nonideal sampling and interpolation from noisy observations in shift-invariant spaces," IEEE Transactions on Signal Processing, vol. 54, no. 7, pp. 2636-2651, July 2006.

[3] M. Unser, "Splines: A perfect fit for signal and image processing," IEEE Signal Processing Magazine, vol. 16, no. 6, pp. 22-38, November 1999, IEEE Signal Processing Society's 2000 magazine award.

[4] L.I. Rudin, S. Osher, and E. Fatemi, "Nonlinear total variation based noise removal algorithms," Physica $D$, vol. 60, no. 1-4, pp. 259-268, November 1992.

[5] J.M. Bioucas-Dias, M.A.T. Figueiredo, and J.P. Oliveira, "Total variation-based image deconvolution: A majorization-minimization approach," in Proceedings of the Thirty-First International Conference on Acoustics, Speech, and Signal Processing (ICASSP'06), Toulouse, France, May 14-19, 2006, pp. II-861-II-864.

[6] D.R. Hunter and K. Lange, "A tutorial on MM algorithms," The American Statistician, vol. 59, no. 1, pp. 30-37(8), February 2004.

[7] M. Nikolova and M.K. Ng, "Analysis of half-quadratic minimization methods for signal and image recovery," SIAM Journal on Scientific Computing, vol. 27, no. 3, pp. 937-966, 2005.

[8] "Brainweb: Simulated MRI volumes for normal brain," McConnell Brain Imaging Centre, www.bic.mni.mcgill.ca/brainweb/selection_normal.html.

[9] C. Voguel, Computational Methods for Inverse Problems, SIAM, Philadelphia, 2002. 